\title{
Eyelash Loss
}

\author{
David R. Jordan, M.D. ${ }^{1}$
}

Loss of eyelashes may occur with several conditions, including a variety of skin diseases, drug side effects, endocrine disorders, metabolic abnormalities, traumatic insults, inflammations, toxins, psychiatric disorders, and systemic disease. If the history and results of clinical, laboratory, and consultative assessments are negative, one must consider the possibility the patient is pulling them out (trichotillomania). Three case reports of spontaneous lash loss are described-one secondary to trichotillomania, one a result of hypothyroidism, and another with features of both hypothyroid and trichotillomania- to illustrate the presentation and appropriate workup to obtain a diagnosis.

KEYWORDS: Lash loss, madarosis, milphosis, allopecia, trichotillomania

There are numerous terms used to describe the isolated loss of eyelashes. Milphosis refers to the falling out of eyelashes. ${ }^{1,2}$ Although a synonymous term often used to describe eyelash loss from any cause, ${ }^{1-3}$ madarosis was originally used to describe eyelash loss resulting from a destructive process. ${ }^{4}$ Madarosis may also be used to describe loss of brow hairs. ${ }^{4}$ Alopecia is a more general term used to describe the absence or loss of hair from any skin area where it is normally present. ${ }^{1,2}$ Alopecia areata is a relatively common condition of undetermined etiology that involves a circumscribed patchy loss of hair in several areas including the scalp, brows, eyelashes, and bearded portion of the face. ${ }^{1,2}$ Alopecia adnata has been used to describe eyelash loss but more appropriately refers to an underdevelopment of the eyelashes rather than a falling out or loss of the eyelashes as in milphosis or madarosis. ${ }^{1}$ The term trichotillomania refers to a rare form of hair/eyelash loss resulting from avulsion of hairs by the patient. There is a compulsive pulling out of one's hair, associated with tension or an irresistible urge before pulling followed by pleasure or relief. ${ }^{1,2,5}$

Loss of eyelashes may occur in numerous conditions, including a variety of skin diseases, endocrinologic disorders, traumatic insults, as well as many others
(Table 1) ${ }^{3-5}$ A careful history documenting the nature of the lash loss as well as any other area of hair loss along with a search for any historical clues indicative of systemic disease is important as a first step to the diagnosis. We report three young patients with spontaneous falling out of eyelashes and review the workup to obtain a diagnosis.

\section{CASE 1}

A 12-year-old child was seen in consultation for loss of lashes involving the left upper lid (Fig. 1). The child was aware he was pulling them but not sure why. He was a good student at school, enjoyed his studies, was not being teased, and seemed to be getting along well at home with his family and friends. He was otherwise well with no systemic illness. On examination multiple broken lashes were noted. There was no sign of blepharitis or other eyelid inflammation. A thyroid-stimulating hormone (TSH) and calcium test were normal. A dermatologic consultation did not reveal any other areas of hair loss or any clue to the etiology of his isolated lash loss. A provisional diagnosis of trichotillomania was made.

\footnotetext{
${ }^{1}$ University of Ottawa Eye Institute Ottawa, Ontario, Canada. Address for correspondence and reprint requests: Dr. 'David R. Jordan, 104-340 McLeod Street, Ottawa, Ontario, Canada K2P 1A4. Ph.D.
}

Semin Plast Surg 2007;21:32-36. Copyright (C) 2007 by Thieme Medical Publishers, Inc., 333 Seventh Avenue, New York, NY 10001, USA. Tel: +1(212) 584-4662.

DOI 10.1055/s-2007-967745. ISSN 1535-2188. 


\section{Table 1 Loss of Eyelashes ${ }^{3-5}$}

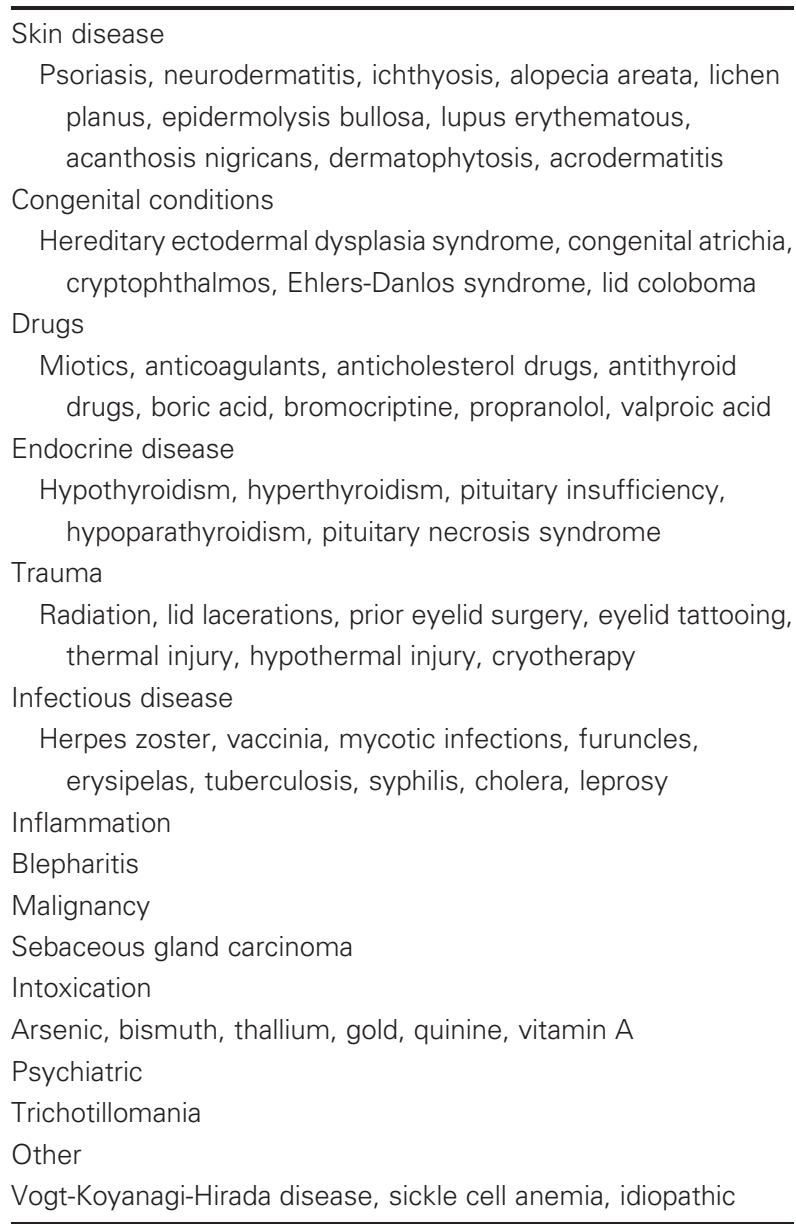

\section{CASE 2}

A 25-year-old woman was seen in consultation for loss of lashes involving all four eyelids (Fig. 2). The patient stated that beginning at age 16, the lashes would spontaneously fall out in some areas, then grow back. She also developed loss of brow hair. She described having an urge to pluck the brows and would pluck them but did not admit to plucking the eyelashes. The

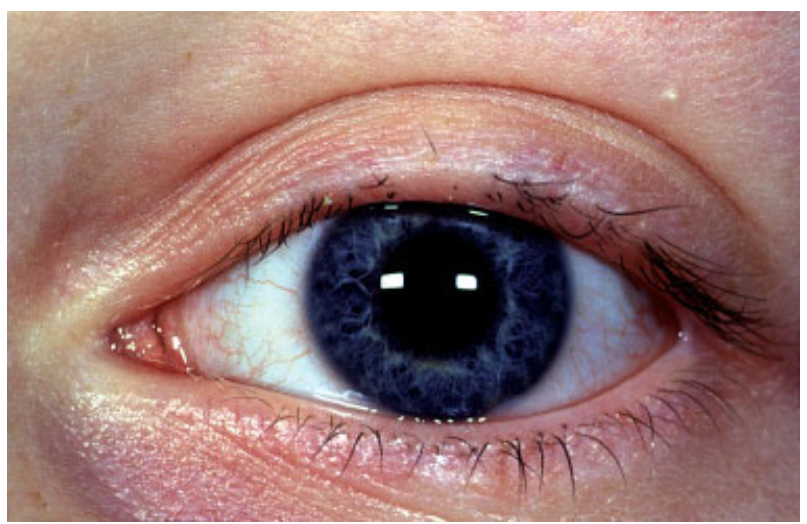

Figure 1 Multiple broken lashes are seen in the central portion of the upper eyelid adjacent to normal lashes.

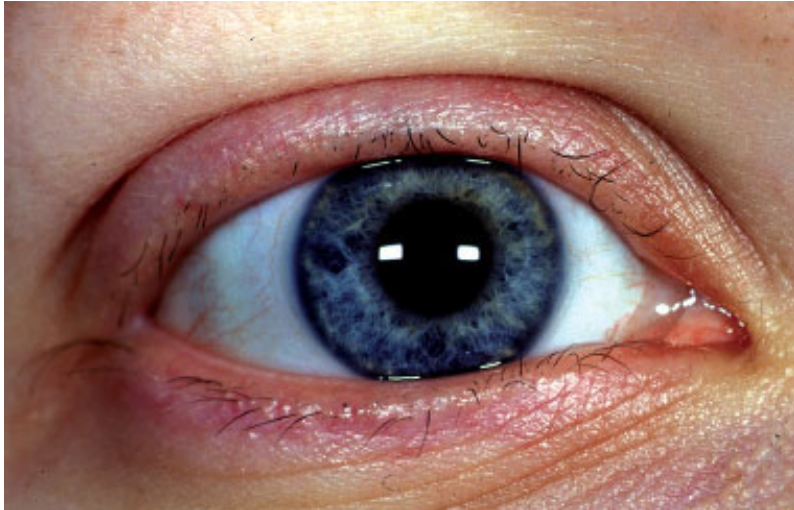

Figure 2 Several areas of broken lashes adjacent to normal lashes on the upper and lower eyelid.

patient was otherwise well, with no known medical illness. She was attending university, doing well, and did not feel stressed at school or home. On examination there were multiple areas on all four eyelids that revealed a loss of lashes. The brow hairs were also very sparse. There was no sign of blepharitis. A dermatologic consultation did not reveal any other areas of hair loss or clues to the diagnosis. Serum calcium was normal; however, serum TSH was slightly high at $5.8 \mathrm{mU} / \mathrm{L}$ (normal $=0.3$ to $4.7 \mathrm{mU} / \mathrm{L}$ ) indicating possible hypothyroidism. The patient was seen by an endocrinologist, who confirmed the slightly high TSH, a low $\mathrm{T}_{4}(13 \mathrm{pmol} / \mathrm{L}$ [normal $=9.1$ to $\left.23.8 \mathrm{pmol} / \mathrm{L}]\right)$, and the presence of antithyroid antibodies. The patient was felt to have a compensated hypothyroidism consistent with Hoshimoto's thyroiditis. Follow up was recommended as she was at risk of becoming more hypothyroid. Unfortunately she did not maintain her follow-up appointment. The mild thyroid dysfunction may have played a role in the lash loss; however, the urge to pull the lashes and failure to maintain follow-up were more suggestive of trichotillomania.

\section{CASE 3}

A 19-year-old woman was seen in consultation for loss of eyelashes involving the right upper lid. The problem began $1 \frac{1 / 2}{2}$ months prior to consultation. The patient described the eyelashes simply falling out spontaneously every 1 to 3 days from the right upper lid. She denied rubbing the lid or pulling the eyelashes. There was no itching or irritation of the eyelid. There was no known skin disease, and she described herself as very healthy except for a mild asthma condition requiring infrequent use of an inhalant. There were no known allergies and no hair loss elsewhere. The patient had recently been stressed because of a high University workload and less than average marks; she was contemplating dropping out of school. She was questioned about pulling her eyelash hairs but denied doing so. A private interview with an 
A
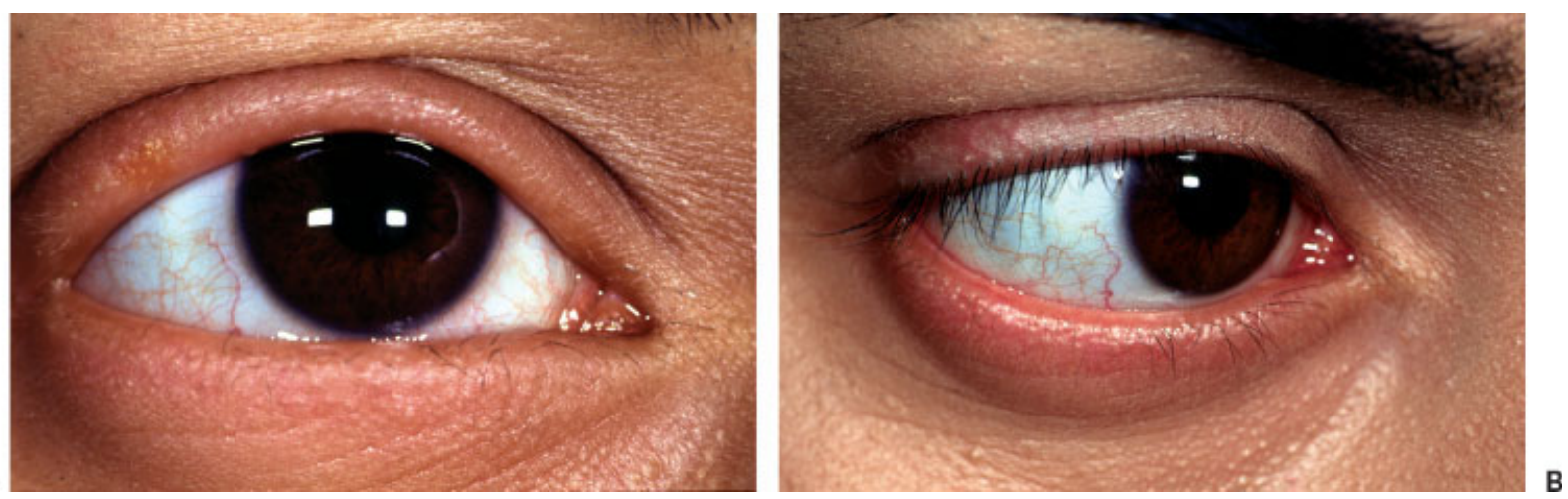

Figure 3 (A) Loss of eyelashes involving entire right upper lid and the lateral two thirds of the lower lid. (B) Nineteen months after treatment of hyperthyroidism, eyelashes have returned on upper lid and are starting to return on lower lid, although they remain sparse.

accompanying relative confirmed a stressful social situation, suggesting the possibility of self-induced eyelash pulling (trichotillomania).

On examination there was loss of eyelashes involving the entire right upper lid. No broken eyelashes or newly growing eyelashes were seen. The eyelid otherwise appeared normal with no sign of eyelid inflammatory erythema, atrophy, or scarring. The remaining ocular exam was unremarkable. Routine thyroid function studies and serum calcium were ordered and a follow-up visit was arranged. The calcium level was normal, whereas thyroid function tests revealed a markedly elevated $\mathrm{T}_{4}$ and low TSH. The patient was seen in follow-up 1 month later with further loss of eyelashes involving the right lower lid (Fig. 3A). There was also a small patch of hair loss involving the left occiput. Further medical history revealed a positive family history for thyroid disease. The patient also reported some mild heat intolerance. An endocrinologic assessment revealed a history of headaches, insomnia, and a large goiter, consistent with her hyperthyroid state. Propylthiouracil as prescribed. Within a month she had gained 10 pounds and her thyrotoxic symptoms (heat intolerance, headache, insomnia) had resolved. Within 3 months of initiating the propylthiouracil, the eyelashes started to grow back and the area of hair loss over the occiput had begun to fill in. Nineteen months later the eyelashes showed continued growth (Fig. 3B) and the patient remained euthyroid. Although trichotillomania was initially suspected, the thyroid dysfunction detected as well as the hair regrowth with treatment confirmed the thyroid as being responsible for the lash loss. The second patch of hair loss was most likely related to the thyroid dysfunction but also raises the possibility of alopecia areata.

\section{DISCUSSION}

When faced with a patient having eyelash loss, a thorough medical history is essential as it may offer clues to the diagnosis. Current or past skin problems, endocri- nologic disorders (hypo- or hyperthyroidism), previous lid infections (herpes zoster), previous lid surgery, other systemic conditions (e.g., lupus), as well as a variety of other conditions all may have eyelash loss associated with them (Table 1). ${ }^{3,5}$ There is a lengthy list of medications that may potentially cause drug-induced hair loss. ${ }^{3}$ It is important to determine the potential side effects of any medication the patient is using. One must examine the eyelids and eyelash roots, searching for clues to the diagnosis (e.g., the collarettes and telangiectasia of blepharitis). Other areas of hair loss are also important to document as in the patients described herein. If no clues are seen clinically, a few laboratory tests such as TSH and calcium level may be helpful to detect an underlying systemic condition (e.g., thyroid dysfunction, hypoparathyroidism) that may potentially have hair loss associated with it. A dermatologic consultation is also beneficial to determine if there are other areas of hair loss or any signs of a skin disorder that may be associated with hair loss. If the history, clinical examination, and laboratory and consultative assessments are negative, one must consider the possibility of trichotillomania. ${ }^{5}$

Patient 1 was healthy with no evidence of dermatologic, endocrinologic, or other evidence of disease and was felt to have trichotillomania. Patient 2 had laboratory evidence of a thyroid dysfunction, which may lead to hair loss, ${ }^{6}$ but also admitted to having an urge to pluck the brow hairs and would pluck them to satisfy this urge (a feature of trichotillomania). She was otherwise well and was thought to have compensated hypothyroidism. No treatment was suggested by the endocrinologist other than follow-up for more overt manifestations of the hypothyroidism. It is therefore difficult to be sure what role the thyroid was playing in the hair loss. The fact that the patient admitted to plucking the brow hairs and the failure to return on follow-up makes one suspect that the lash loss was due to trichotillomania rather than compensated hypothyroidism.

The third patient reported herein was initially felt to be medically well by history but was under a lot of stress. Trichotillomania was contemplated on the first 
consultative visit as there were no clues to the diagnosis of the eyelash loss. Trichotillomania is more common in women, and the most common site for hair pulling is the central region of the scalp followed by the eyebrows and eyelashes. ${ }^{5}$ The patients generally present with a history of eyelashes falling out, as in our patient, with no clear explanation. The hair/eyelash loss in trichotillomania may be incomplete with short, broken hairs (as in case 1) and some long, normal-appearing hairs. ${ }^{7}$ The diagnosis of trichotillomania may be difficult to confirm, as compulsive hair pullers frequently conceal or deny their habit. ${ }^{8}$ Hair pulling may be precipitated by a stressful event such as parental divorce, death of a relative, or studying for a test. ${ }^{9}$ The elevated $\mathrm{T}_{4}$, low TSH level, history of heat intolerance, insomnia, headaches, goiter, and a positive family history for thyroid disease in our patient confirmed the diagnosis of hyperthyroidism and made trichotillomania less likely as a diagnostic possibility.

Thyroid hormone influences the activity of all cells in the mammalian body. It is therefore not unexpected that hair follicle activity is affected in pathologic states such as hypothyroidism or hyperthyroidism. ${ }^{10}$ In patients with hyperthyroidism, hair changes have been described as thinning with breaking off and shortening of the hair, as well as patchy areas of hair loss (alopecia). ${ }^{4,11,12}$ In hypothyroidism the hair may become dull, brittle, and coarse with reduced diameter as well as areas of hair loss that may involve the eyelashes and brows., $90,13-15$

Although these effects on human hair loss have been known for generations, it is only in recent years that thyroid hormone receptors have been localized on hair follicle cells, indicating that thyroid hormones may affect hair growth and the hair cycle directly rather than simply through an intermediate mechanism such as the patient's general metabolic state. ${ }^{16,17}$ Changes of hair growth and hair structure may potentially be the first clinical sign of a thyroid hormonal disturbance as a result of the influence of the thyroid hormone on the cell cycle kinetics of the hair follicle cells. ${ }^{6}$

The second patch of hair loss in patient 3 involving the scalp, in addition to the eyelash loss, raised the possibility of alopecia areata. Alopecia areata usually causes a circumscribed unscarred loss of hair, typically involving the scalp or bearded portion of the face. ${ }^{4,18-20}$ It is characterized by the sudden appearance of bald patches often in apparently normal persons without obvious local cause or systemic disease. ${ }^{18,19}$ Eyelash loss as the initial manifestation of alopecia areata may occur but is uncommon. ${ }^{4}$ Patients with trichotillomania may initially be diagnosed as having alopecia areata. ${ }^{5}$ The course of alopecia areata is unpredictable; the hair in a bald patch may regrow in a few months, fresh patches may appear, or all the hair on the head and body may be lost (alopecia universalis). Alopecia areata is a relatively common disorder but the cause remains unknown. ${ }^{18,19}$ An increased incidence of circulating organ-specific antibodies as well as its coexistence with other autoimmune disorders and the presence of lymphocytic infiltration of the involved hair follicles suggest that an immunological mechanism may be involved in the pathogenesis of the disease. ${ }^{1,21}$ There is an increased incidence of alopecia areata in patients with other autoimmune disorders including diabetes mellitus, thyroid disease, vitiligo, pernicious anemia, and Addison's disease. , $^{5,19}$

It is difficult to be certain whether the third patient reported herein had eyelash loss (and subsequent scalp hair loss) as the initial sign of alopecia areata occurring simultaneously with hyperthyroidism or whether the eyelash loss and scalp hair loss were early signs of a hyperthyroid state due to a direct effect of the increased thyroid hormone levels on the cell cycle kinetics of the eyelash hair follicles (which are known to have thyroid hormone receptors on their surface). It is also possible, although more unlikely, that she was epilating her eyelashes but denying it (trichotillomania). Collecting hairs and examining the roots may have been helpful in differentiating alopecia areata from other diseases causing hair loss such as trichotillomania. ${ }^{1,5,22}$ The anagen or growing hair root found in trichotillomania is easy to distinguish from the normal telogen or resting hair root. Scalp hairs that have been epilated usually show 80 to $90 \%$ in anagen (growing) and 10 to $20 \%$ in telogen (resting) phase. Approximately 100 scalp hairs are shed daily in the normal scalp and $100 \%$ of the roots are usually in telogen. If the patient had alopecia areata, examination of shed hairs would also have shown no visible hair root but a tapered proximal end produced by the interruption of the anagen phase of the cycle. ${ }^{19,22}$ Skin biopsy may also help differentiate alopecia areata from trichotillomania. The characteristic features of trichotillomania on skin biopsy include plugging of the empty follicles with heavily pigmented, soft keratinous material and a varying degree of atrophy. In alopecia areata one sees an inflammatory response on the growing hairs. ${ }^{6}$

In summary, hair loss involving the eyelashes may result from a variety conditions (Table 1 ). The patients generally present with a history of lashes falling out with no clear explanation. Careful history taking, searching for evidence of skin diseases in the past or present, systemic disease, drug side effects, depression or recent severe stress, and physical examination for broken hairs, signs of lid trauma, eyelid inflammation, and so on may provide helpful clues. A dermatologic consultation may be useful, as there might be other areas of hair loss or skin disease that provide a clue to the underlying problem. A few simple laboratory tests (serum calcium, TSH) may be helpful to rule out other reasons for hair loss such as endocrine abnormalities. Eyelash loss as the presenting sign of thyroid dysfunction is uncommon. The diagnosis, however, can be facilitated by confirming symptoms and signs of thyroid disease as well as abnormal thyroid function tests (case 3). When no clear explanation exists 
for the lash loss and multiple areas of broken lash stubble are noted, self-induced lash pulling (trichotillomania, the irresistible urge to remove one's hair), although uncommon, should be considered (cases 1 and 2).

\section{REFERENCES}

1. Barlow-Pugh M, ed. Stedmans Medical Dictionary. 27th ed. Philadelphia, PA: Lippincott Williams \& Wilkins; 2000

2. Anderson DM, ed. Dorlands Illustrated Medical Dictionary. 29th ed. Philadelphia, PA: W.B. Saunders; 2000

3. Roy F, ed. Ocular Syndromes and Systemic Diseases. New York: Grune \& Stratton; 1985:115-117

4. Duke-Elder S, ed. System of Ophthalmology. The Ocular Adnexa Part I-Diseases of the Eyelashes. London: Henry Kimpton; 1974:377-382

5. Mawn L, Jordan DR. Trichotillomania. Ophthalmology 1997; 104:2175-2178

6. Schell H, Kresewetter F, Seidel C, Hintzenstern JV. Cell cycle kinetics of human anogen scalp hair bulbs in thyroid disorders determined by DNA flow cytomy. Dermatologica 1991;182:23-26

7. Mehregan A. Trichotillomania: a clinicopathological study. Arch Dermatol 1970;102:129-133

8. Swedo S, Rappaport J. Annotation: trichotillomania. Journal of Child Psychology and Psychiatry 1991;32:401-409

9. Graber J, Arndt W. Trichotillomania. Comp Psychiatry 1993;34:340-346

10. Comaish JS. The thyroid and hair growth. Semin Dermatol 1985;4:4-8
11. Olufunsho O, Bella F, Bella AF. Thyrotoxiosis in Nigeria: analysis of a five year experience. Topical and Geographical Medicine 1990;42:248-254

12. Rook A. Endocrine influences on hair growth. BMJ 1965; 1:609-614

13. Mahto RS. Ocular features of hypothyroidism. Brit J Ophthal 1972;56:546-549

14. Kingery FA. Eyebrows, plus or minus. JAMA 1966;195:7: 571

15. Wahlberg J. Hypophysectomy adiposity and emaciation. JAMA 1936;106:1968-1970

16. Messenger AG. Thyroid hormone and hair growth. Br J Dermatol 2000;142:631-635

17. Billoni N, Buan B, Gauthier B, Gaillard O, Mahe YF, Bernard BA. Thyroid hormone receptor $\mathrm{B}_{1}$ is expressed in the human hair follicle. Br J Dermatol 2000;142:645652

18. Beers MH, Berkow R, eds. Alopecia. In: The Merck Manual of Diagnosis and Therapy, 17th ed. Whitehouse Station, NJ: Merck Research Laboratories; 1999:814-815

19. Orentreich DS, Orentreich N. Dermatology of the eyelids (excluding neoplasms). In: Della Rocca RC, Nesi FA, Lismar RD, eds. Byron C Smith Ophthalmic Plastic and Reconstructive Surgery Volume 2, St. Louis: CV Mosby; 1987: 855-906

20. Offret H, Venencie PY, Gregoire-Cassoux N. Madarose et palade des cils. J Fr Ophthalmol 1994;17:486-488

21. Safai B, Orentreich N, Good RA. Immunological abnormalities in patients with alopecia areata. Clin Res 1979;27: $244 \mathrm{~A}$

22. Orentreich N. Hair loss in a young boy: trichotillomania or alopecia areata. JAMA 1975;234:761 